\title{
Clinical and molecular evaluation of 16 patients with Rett syndrome
}

\author{
Pınar Zengin-Akkuş ${ }^{1}$, Ekim Z. Taşkıran², Serkan Kabaçam ${ }^{1}$, Pelin Özlem Şimşek-Kiper ${ }^{1}$, \\ Göknur Haliloğlu ${ }^{3}$, Koray Boduroğlu${ }^{1}$, Gülen Eda Utine ${ }^{1}$ \\ Division of ${ }^{1}$ Pediatric Genetics and ${ }^{3}$ Neurology, Department of Pediatrics and ${ }^{2}$ Medical Genetics, Hacettepe University \\ Faculty of Medicine, Ankara, Turkey. \\ E-mail: pinarzengin@hacettepe.edu.tr \\ Received: 6th June 2017, Revised: 12th July 2017, Accepted: 13th August 2017
}

SUMMARY: Zengin-Akkuş P, Taşkıran EZ, Kabaçam S, Şimşek-Kiper PÖ, Haliloğlu G, Boduroğlu K, Utine GE. Clinical and molecular evaluation of 16 patients with Rett syndrome. Turk J Pediatr 2018; 60: 1-9.

Rett syndrome is a neurodevelopmental disorder caused by mutations in MECP2. The disease is characterized by early neurological regression following a normal initial development. The diagnosis is a clinical one, based on major and minor diagnostic criteria. This study, in a group of patients from a single tertiary center, aimed to evaluate the efficiency of clinical diagnosis and to see if there was a diagnostic delay. A second aim was to investigate genotype-phenotype correlations, based on Pineda scores. In this study, sixteen patients with a median age of 6.5 years (2.5-22 years) were included, following molecular confirmation of clinical diagnosis. The median age at the onset of symptoms and the median age at clinical diagnosis was 1.5 years and 2.5 years, respectively, the difference being statistically significant. Considering the Rett syndrome diagnostic criteria, initially regulated in 2002 and revised in 2010, seven and two patients in our group, respectively, did not meet the main criteria. Pineda scores among mutation groups were statistically not different. To conclude, the present study revealed presence of a diagnostic delay. The challenge may be that the patients do not exhibit full-blown clinical picture initially. No genotype-phenotype correlations were detected in clinical severity, as measured by Pineda scores. Moreover, the diagnostic criteria revised in $\mathbf{2 0 1 0}$ are more comprehensive as compared to the 2002 criteria; however, further revision may increase diagnostic sensitivity.

Key words: Rett syndrome, MECP2, diagnostic delay, genotype-phenotype correlation.

Rett syndrome is a neurodevelopmental disorder affecting exclusively females. This X-linked disease affects an estimated 1 in 10,00015,000 females. ${ }^{1}$ It is characterized by early neurological regression following normal initial development. Regression almost always affects cognitive, verbal, fine and gross motor abilities, and communication. Autonomic dysfunction and seizures usually accompany. ${ }^{2}$ The diagnosis is on clinical grounds owing to the unique developmental profile, however, other diseases may present with overlapping features and therefore, differential diagnosis may sometimes be challenging. Besides, clinically recognized stages of Rett syndrome, as well as presence of atypical forms, may further complicate the diagnosis. ${ }^{3}$

Clinical diagnostic criteria for Rett syndrome were established in 2002.4 With better understanding of the clinical characteristics, these criteria were revised in 2010 by Neul et al. ${ }^{3}$ Scoring systems for assessment of clinical severity have also been developed for Rett syndrome. ${ }^{5-8}$ Pineda scoring system developed by Monros et al. 8 in 2001 mainly includes parameters that are independent from the timing of scoring, such as the presence and time of onset for microcephaly, unsupported sitting, ambulation, language, epilepsy, respiratory 
function, hand use and stereotypies.

Mutations in MECP2 gene were identified in 1999 as the molecular cause of Rett syndrome. ${ }^{9}$ MECP2 gene is located in Xq28 and encodes for methyl-CpG binding protein (MBD). DNA analysis for mutations is only supportive for the diagnosis. Presence of mutations is by no means diagnostic, since there are other MECP2-related disorders. ${ }^{3,10}$ Mutations are detected in $95 \%$ of patients with classic Rett syndrome and in $75 \%$ of patients with atypical Rett syndrome. There are more than a thousand MECP2 mutations associated with Rett syndrome ${ }^{7}$, however, R106W, R133C, T158M, R168X, R255X, R270X, R294X, R306C and C-terminal truncating mutations are the most common. These eight point mutations are responsible for nearly $70 \%$ of cases. ${ }^{11}$ Data in literature implies presence of genotype-phenotype correlations, however, no clear results are achieved yet. ${ }^{12}$

The current study was intended to document clinical and molecular features of patients with Rett syndrome, to investigate whether current diagnostic criteria cover all patients efficiently and to search for potential genotype-phenotype correlations.

\section{Material and Methods}

\section{Patients}

Sixteen consecutive patients diagnosed clinically with Rett syndrome at Hacettepe University Faculty of Medicine, Department of Pediatric Genetics, and in whom MECP2 mutations were shown in peripheral blood DNA analysis were included in the study. The study protocol was approved by Hacettepe University Medical Faculty Ethical Committee in March 2014. (GO 14/87-13). All patients diagnosed with Rett syndrome in our clinic at the time of this study were included. Patient data were collected from past medical records. The age at diagnosis was accepted as the first time a physician considered Rett syndrome as a diagnostic possibility. Time lag between the first parental concern and the first suspicion of the clinical diagnosis was considered as "diagnostic delay".Patients who met diagnostic criteria but did not possess MECP2 mutations were excluded from the study.

Patient data on perinatal and postnatal history, developmental milestones, physical and neurological examination findings were gathered. All patients were scored according to Pineda scoring system. ${ }^{8}$ Patients were scored with Pineda system in order to determine the clinical severity, and to evaluate any possible genotype-phenotype correlations. Patients were first grouped into $M E C P 2$ gene regions where their mutations reside. Then, a second grouping was made based on the mutation types; as missense or truncated, the latter including nonsense and frameshift mutations. Pineda scores were compared among groups of mutation site and also among groups of mutation types.

\section{Molecular analysis}

DNA from peripheral blood lymphocytes were obtained using The QIAamp DNA Blood Mini Kit (Qiagen) according to manufacturer's recommendations. Sanger sequencing was performed using BigDye terminator chemistry 3.1 on the 3130 Genetic Analyzer (Applied Biosystems). Primer sequences and PCR conditions are available on request. Multiplex ligation-dependent probe amplification (MLPA) was done for detecting copy number changes in MECP2 gene and flanking L1CAM, IRAK1, SYBL1 gene exons. MLPA was done using SALSA MLPA ${ }^{\circledR}$ probemix P015C kit (MRCHolland, Amsterdam, Netherlands) and was analysed using Coffalyser ${ }^{\circledR}$ program.

\section{Statistical Analysis}

Statistical analyses were performed with SPSS (Statistical Package for the Social Sciences (SPSS) for Windows 18.0). A Wilcoxon signedrank test or Mann-Whitney U test, when appropriate, was used to compare differences between the groups. Statistical tests were considered to be significant when $\mathrm{p}<0.05$.

\section{Results}

The study group consisted of 16 female patients (Table I) with a median age of 6.5 years (range; 2.5-22 years). Initial admission to a physician with a suspicion of abnormal development and subsequent assessment by a child neurologist (Fig. 1 and Table I) were both at the median age of 1.5 years (range; 8 months- 4 years). However, median age of admission to the Department of Genetics was at 3 years of age (range; 14 months-17 years).

All patients were clinically consistent with Rett 


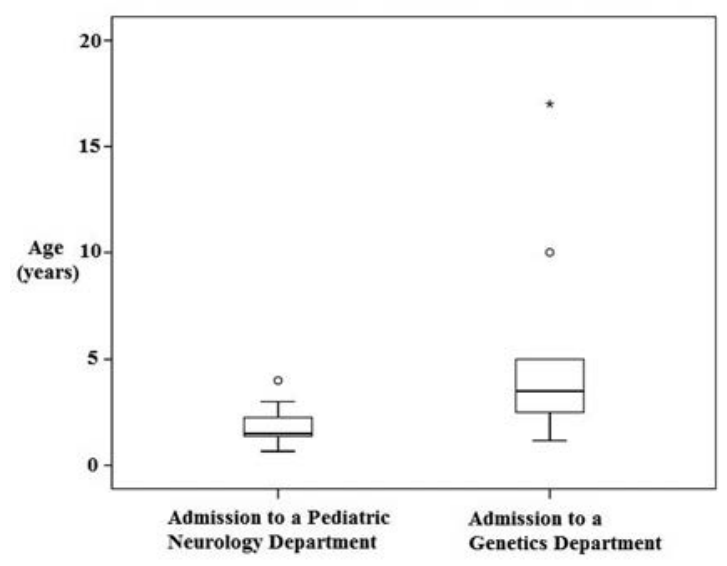

Fig. 1. Age at admission to Departments of Pediatric Neurology and Genetics

syndrome and were shown to bear MECP2 mutations later on. The age at diagnosis ranged between 10 months and 5 years (median; 2.5 years). Diagnostic delay ranged from 0 to 3.5 years (median duration; 1 year). The time difference between first parental concern and clinical diagnosis was statistically significant $(p<0.05)$. Rett syndrome was considered during the first visit for only three of the patients; these patients with no diagnostic delay were 1.5, 1.5 and 2 years old (patients 5, 6 and 13; Table I). Timing for MECP2 mutation analysis ranged from 1.5 to 21 years of age (median; 5 years), however, this was not taken into account in assessing diagnostic delay, since laboratory facilities available for patients were not standard and testing was delayed due to various factors.

The patients were clinically diagnosed based on the diagnostic criteria created in 2002 and revised in 2010.3,4 Seven of sixteen patients did not meet the criteria created in 2002 and could not be clinically diagnosed. Three of these seven patients (number 4, 6 and 14) had no microcephaly. Therefore, they were assumed not to meet the postnatal deceleration of head growth criteria in spite of the lack of the data about head circumference at birth. Four patients (patient number 2, 3, 11 and 12) did not meet criteria on loss of achieved purposeful hand skills between ages $1 / 2-21 / 2$ years. Patient 2 had never achieved purposeful hand skills. Other three patients, numbers 3,11 , and 12 , lost previously achieved purposeful hand skills at the ages of 5, 3 and 9 years, respectively.

On evaluation according to the criteria revised

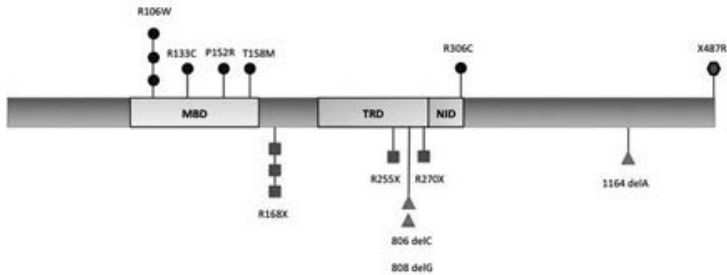

Fig. 2. A schematic illustration of $M E C P 2$ representing the major functional domains. The mutations of MECP2 detected in the study are depicted according to their location in the protein. Each symbol represents a single case with a MECP2 mutation. Circle, square, triangle and hexagon represents missense, nonsense, frameshift and stop-loss mutation respectively (MBD; Methyl-CpG binding domain, TRD; Transcription repression domain, NID; nuclear receptor interaction domain).

in $2010^{3}$, it was observed that two patients did not meet all four main criteria (number 2 and 11). Parents of the first patient (number 2) were not aware of any purposeful hand use. Therefore, this patient did not meet the criteria on partial or complete loss of acquired purposeful hand skills. The other patient's (number 11) parents considered her development as abnormal comparing with her siblings in first six months and abnormal psychomotor development during the first six months of life is among the exclusion criteria. Patient number 11 did not have a history of perinatal hypoxia and her cranial magnetic resonance imaging was normal. We consider these two patients had Rett syndrome based on previously reported typical mutations and clinically consistent features as shown in Table I. All other patients had typical Rett syndrome according to the 2010 criteria.

Detailed clinical and molecular genetic backgrounds of the patients are summarized in Table I. Microcephaly was absent in three patients and psychomotor development during the first six months was not normal in one. Three patients aged 9, 4 and 10 had no stable periods but continuous progression, while the rest had stable periods between 2.5-11 years of age (median; 3.2 years).

All patients had severe deterioration in communication skills, psychomotor retardation and stereotypic hand movements, whereas Patient 2 had never acquired purposeful hand movements. Loss of purposeful hand movements was later than 30 months in 3 patients. Twelve patients completely lost speech, while 4 had some residual words. Stereotypic 


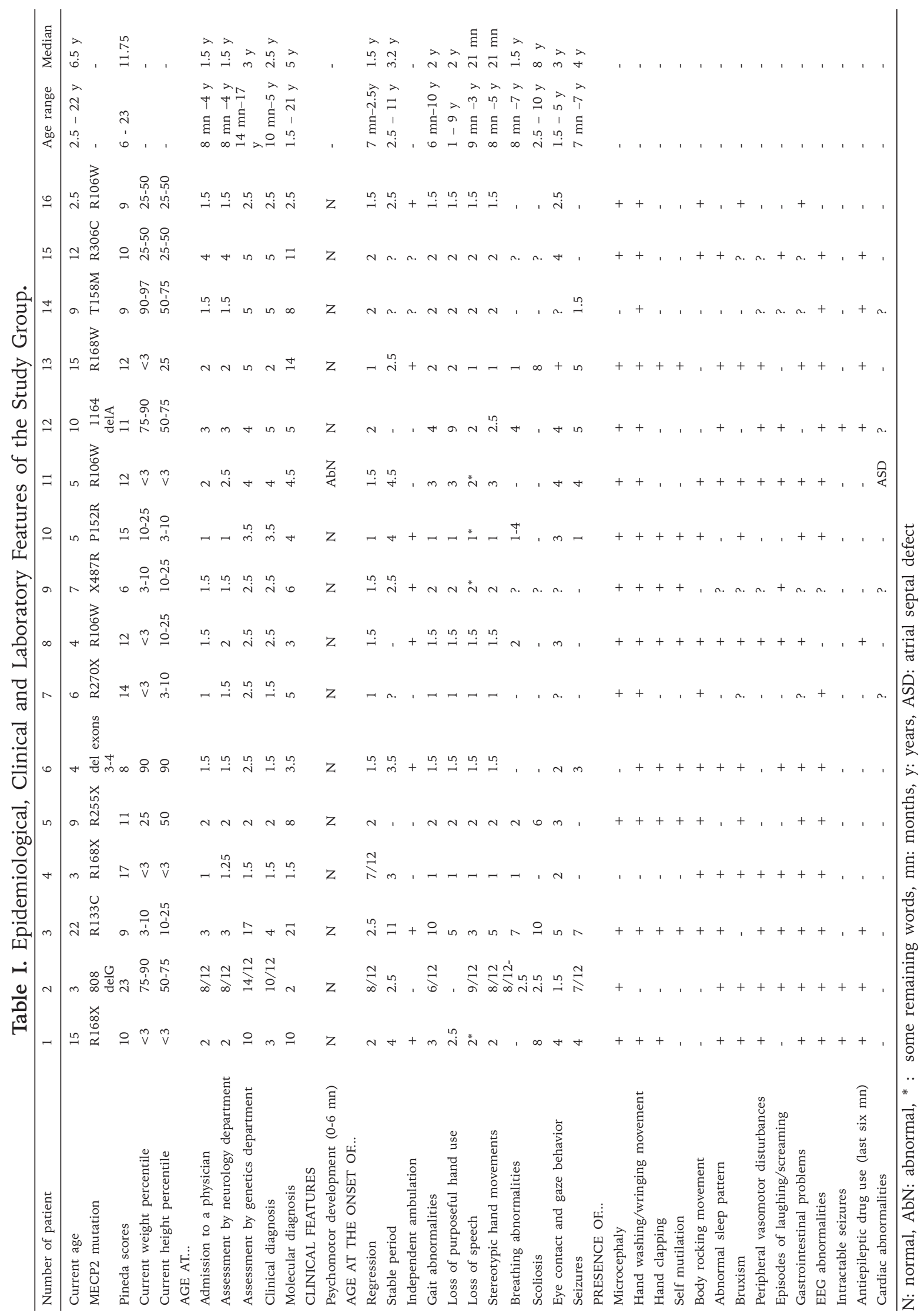


Table II. Mutation Types, Mutation Regions and Pineda Scores of Patients.

\begin{tabular}{|c|c|c|c|}
\hline Mutation region & $\begin{array}{l}\text { Mutations (mutation } \\
\text { types) }\end{array}$ & Patient numbers & $\begin{array}{l}\text { Pineda Scores respectively } \\
\text { (Median) }\end{array}$ \\
\hline $\mathrm{MBD}^{*}$ & $\begin{array}{l}\text { R106W (missense) } \\
\text { R133C (missense) } \\
\text { P152R (missense) } \\
\text { T158M (missense) }\end{array}$ & $\begin{array}{l}8,11,16 \\
3 \\
10 \\
14\end{array}$ & $\begin{array}{l}12,12,9 \\
9 \\
15 \\
9 \\
(10.5)\end{array}$ \\
\hline $\mathrm{TRD}^{* *}$ & $\begin{array}{l}\text { 808delG (frameshift) } \\
\text { R255X (nonsense) } \\
\text { R270 (nonsense) } \\
\text { R306C (missense) }\end{array}$ & $\begin{array}{l}2 \\
5 \\
7 \\
15\end{array}$ & $\begin{array}{l}23 \\
11 \\
14 \\
10 \\
(12.5)\end{array}$ \\
\hline Interdomain & R168X (nonsense) & $1,4,13$ & $\begin{array}{l}10,17,12 \\
(12)\end{array}$ \\
\hline C-Terminal & $\begin{array}{l}\text { X487R (non-stop) } \\
\text { 1164delA(frameshift) }\end{array}$ & $\begin{array}{l}9 \\
12\end{array}$ & $\begin{array}{l}6, \\
11 \\
(8.5)\end{array}$ \\
\hline Exon $3-4$ & Deletion of exon3-4 & 6 & $\begin{array}{l}8 \\
(8)\end{array}$ \\
\hline
\end{tabular}

MBD: methyl-CpG binding protein, TRD: transcription repression domain

hand movements were observed in all, while Patient 16 interestingly had midline hand movements more frequent on the posterior. Ten patients had stereotypic body rocking movements; back and forth or left and right. Two patients had shivering, and 4 had head shaking. Abnormal mouth movements such as opening and closing of lips, smacking of the mouth were present in 5 patients. Seven patients had self-mutilation behaviour such as hand biting or head knocking. Apart from these stereotypies, patients also had stereotypic movements such as hip lifting and spitting.

Growth retardation was detected in 6 patients, while height and weight were above third percentile in ten. Five patients developed scoliosis between 2.5 and 10 years (median; 8 years), while patients without scoliosis were aged 3 to 10 years (median; 5 years). None of the patients was reported to have a history of decreased response to pain. Various gastrointestinal problems in 11 patients included constipation, difficulty in swallowing, flatulence and gastroesophageal reflux. Small atrial septal defect and patent foramen ovale were detected in one patient, while none had arrhythmia on Holter monitoring.

MECP2 mutations, shown in Table I and Table II, were classified according to affected protein domains and are depicted in Figure 2. All identified mutations were previously reported and are present in the Rett syndrome database. ${ }^{13}$ Three patients had R106W, one had R133C, one patient had T158M and another one had R152R missense mutation, all of which are located in MBD region. R168X mutation was found in three patients in interdomain region, which is a nonsense mutation. In transcription repression domain (TRD) region, R255X and R270X nonsense mutations were detected in two separate patients. In NID (nuclear receptor interaction domain) of TRD region, a missense R306C mutation was found. There was another 808delgG mutation in TRD region in one patient leading to frame shift. In C-terminal region, 1164delA heterozygous frame shift mutation and X487R mutation were found in two separate patients. Another patient had deletion of exons 3 and 4 .

Pineda scores of patients are also shown in Table I and Table II. Pineda scores of the patients ranged between 6 and 23 (median; 11.75). Scores were compared among mutation groups and ranged as follows: MBD; 9 to 15 (6 patients, median; 10.5), interdomain; 10 to 17 (3 patients, median; 12), TRD; 10 to 23 (4 patients, median; 12.5), C-terminal region; 6 and 11 ( 2 patients, mean 8.5). The score was 8 for the patient with deleted exons 3 
and 4. Pineda scores and mutation types of patients are shown in Table II. A threshold severity score that would possibly predict the mutation domain or provide a clinical severity classification could not be detected statistically. Participation involved informed consents.

\section{Discussion}

Rett syndrome is among the challenging clinical diagnoses owing to features overlapping with other neurodevelopmental diseases. ${ }^{14,15} \mathrm{~A}$ group of etiologically heterogeneous diseases may actually be causative for some features observed in Rett syndrome. Timely clinical recognition of Rett syndrome would enable useful counselling regarding the prognosis, future manifestations, and genetic aspects. A timely diagnosis would also prevent nonyielding diagnostic investigations and prevent further financial and psychological burden to the family. ${ }^{16}$

Our data revealed that patients with Rett syndrome consulting a physician for the first time was immediately referred to Department of Pediatric Neurology, however, they were admitted to the Department of Genetics later. Median delay in diagnosis was one year and this was statistically significant $(p<0.05)$. The present study shows that there is a significant diagnostic delay for patients with Rett syndrome. Challenging factors in this cohort seem to be; a) patients may not fully exhibit core clinical features initially, and b) acquired microcephaly may be absent.

The disease has a characteristic evolution and certain stages. In early stages of the diseases core clinical symptoms may not be fully developed in patients. Microcephaly, developmental delay, regression and autistic features are nonspecific, and have huge differential diagnosis lists. ${ }^{14,15,17,18}$ Initial complaints of stagnation and then regression, along with slowing of the head growth, should suggest a smaller group of several disorders, Rett syndrome being among the most common ones $^{1}$ In the study of Tarquinio et al. ${ }^{19}$, patients with delay in motor skills were diagnosed earlier than the patients who lost acquired skills. Patients without microcephaly and/or decrease in head growth had delays in diagnosis, since all patients with Rett syndrome may not necessarily have acquired microcephaly. ${ }^{19}$
For timely diagnosis and avoidance of unnecessary diagnostic tests, Rett syndrome ought to be kept in mind as a possibility, since apart from the progressive course of the disease, under recognition by physicians may also cause the diagnostic delay. Tarquinio et al. ${ }^{19}$ revealed that pediatricians diagnose minority of Rett syndrome cases and usually not in the early stage. Since these patients may consult a physician for different causes, awareness of pediatricians, general practitioners, child psychiatrists, pediatric neurologists and other specialists would be helpful. Previous literature reports that parents are concerned about early symptoms such as developmental delay, inactivity, lack of interest and being "easy to satisfy". ${ }^{20}$ Fehr et al. ${ }^{21}$ reported that most of the parents were concerned about unusual behaviors or development during infancy. Abnormal hand/body movements and facial expressions may appear in pre-regression period. ${ }^{22}$ Patients may exhibit autistic behavior during early developmental period, as a Danish study ${ }^{20}$ revealed that nearly a third of Rett syndrome girls was not diagnosed, although they had autistic features or behavioral changes. Patient 5 in our group was diagnosed at the age of two, when she was admitted with behavioral changes to the Child Psychiatry Department. Accordingly, Bisgaard et al. ${ }^{20}$ reported that the diagnostic rate of Rett syndrome was nearly doubled after establishment of the National Centre for Rett syndrome in Denmark. For ensuring prompt diagnosis, MECP2 gene analysis is recommended in evaluation of undiagnosed girls with global developmental delay and intellectual disability after performing chromosomal microarray, metabolic tests and fragile $\mathrm{X}$ testing. ${ }^{20,23}$

Our cohort was evaluated in terms of the diagnostic criteria created in 2002 and revised in 2010.3,4 Among the 7 patients who did not meet the 2002 criteria absence of microcephaly in three (patients 4, 6 and 14) and absence of the early loss of achieved purposeful hand skills in four (patients 2, 3, 11 and 12) were evident. Likewise, patients 2 and 11 did not meet the 2010 criteria. Nevertheless, five patients that could not be diagnosed with criteria from 2002 could be diagnosed following the 2010 criteria. However, as molecular analysis became widely available and a better understanding of the disease is achieved, several 
studies revealed that early development might be abnormal in Rett syndrome $20,22,24,25$, and abnormal early development should not be taken as an exclusion criteria. Although our study revealed that criteria revised in 2010 were more comprehensive as compared to the 2002 criteria, further revision may be helpful in increasing the sensitivity of the diagnostic criteria.

Our cohort demonstrated once again that there are also late-onset features like scoliosis and seizures. There were 9 patients without scoliosis, with a median age of 5 years. Ager et al. ${ }^{26}$ has shown that median age for onset of scoliosis was 9.8 years and that only $25 \%$ of the patients who were 6 years-old had scoliosis, this ratio increasing to $75 \%$ by age 13. Similarly, clinical seizures were absent in seven patients in the present group. Seizures usually start after 2 years of age and prevalence increases with age ${ }^{27}$, therefore, follow-up for seizures and/or EEG abnormalities is required.

R106W, R133C, T158M, R168X, R255X, R270X, R294X, R306C mutations in MECP2 were reported to be most frequent in patients. ${ }^{11}$ Consistently, R168X, R106W, R133C, R270X, R255X, T158M and R306C mutations were found in our patient group. One of the common mutations, R294X, was not detected in our cohort.

Previous genotype-phenotype correlation studies revealed variable results. Mutations in the MBD region were associated with more severe disease by Weaving et al. ${ }^{28}$ However, in another study, there was no difference in clinical severity between MBD and TRD groups. ${ }^{29}$ Clinical course was found to be milder with missense mutations as compared to truncating mutations. ${ }^{8,30}$ Besides, course was even milder in late truncating mutations compared to early. ${ }^{30}$

In the study conducted by Neul et al. ${ }^{9}$, which consisted 245 females with Rett syndrome, patients with R133C mutations had milder courses, as compared to R168X mutation and large deletions. With R168X mutation, the disease had a more severe course; walking, purposeful hand use, and speech were more severely affected compared to the R294X mutation and late $\mathrm{C}$-terminal truncating mutations. Ambulation and communication were more likely protected in C-terminal truncating mutations. Speech was more severely affected by R306C, although clinical course was usually milder. ${ }^{11}$ In our group, clinical severity score of the patient with R133C was 9. Scores of patients 1, 4, 13 with R168X were 10, 17 and 12 , respectively. Clinical score of patient 15 with R306C was 10 and spoken words were completely lost. These scores were comparable to those from the study by Neul et al. ${ }^{9}$

Limitations of the study are the small number of patients and absence of XCI studies. We compared Pineda scores across the gene domains harbouring the mutations, and did not detect any statistically significant differences $(p>0.05)$. Similarly, there was no statistically significant difference between scores from missense and truncating mutation groups $(p=0.6313)$, although the score in missense group being lower than the other was consistent with the previous literature. Absence of any genotypephenotype correlations is most probably related to low number of patients in our cohort. Besides, existence of genotype-phenotype correlations may probably be associated not only with the site and type of mutations but also with many other biological mechanisms such as X-chromosome inactivation (XCI) profile and the like. In a study conducted by Amir et al. ${ }^{6}$, random XCI was found in $91 \%$ of patients with Rett syndrome. There are also controversial studies. ${ }^{31}$ XCI pattern may vary between neurons and blood, and thus, establishing a correlation between the ratio of XCI in leukocytes and the severity of the disease is difficult and useless. ${ }^{32}$ Nevertheless, since it was previously reported that skewing in XCI might lead to a milder phenotype even with an early truncating mutation, it is prudent to take into consideration the $\mathrm{XCI}$ pattern in assessing clinical severity. Detection of genotype-phenotype correlations might be temporally difficult, owing to the progressive course of the disease.

This study revealed the presence of a diagnostic delay in Rett syndrome patients and absence of genotype-phenotype correlations regarding Pineda scores. 2010 criteria cover patients more efficiently, however, may be further revised to cover other Rett patients with typical MECP2 mutations. Tarquinio et al. ${ }^{19}$ claims that recognition of the disease by pediatricians has improved, however median age of diagnosis did 
not improve due to lack of systematic effort. As the awareness on the disease will improve, pediatricians would more likely recognize the phenotype and refer patients for genetic testing. Recognizing early developmental problems, atypical features, behavioural changes and suspecting Rett syndrome more frequently may further improve median age of diagnosis.

\section{Acknowledgement}

We would like to thank the families for participating in this study.

\section{REFERENCES}

1. Hagberg B. Rett's syndrome: prevalence and impact on progressive severe mental retardation in girls. Acta Paediatr Scand 1985; 74: 405-408.

2. Dolce A, Ben-Zeev B, Naidu S, Kossoff EH. Rett syndrome and epilepsy: an update for child neurologists. Pediatr Neurol 2013; 48: 337-345.

3. Neul JL, Kaufmann WE, Glaze DG, et al. Rett syndrome: revised diagnostic criteria and nomenclature. Ann Neurol 2010; 68: 944-950.

4. Hagberg B, Hanefeld F, Percy A, Skjeldal O. An update on clinically applicable diagnostic criteria in Rett syndrome. Comments to Rett Syndrome Clinical Criteria Consensus Panel Satellite to European Paediatric Neurology Society Meeting, Baden Baden, Germany, 11 September 2001. Eur J Paediatr Neurol 2002; 6: 293-297.

5. Kerr AM, Nomura Y, Armstrong D, et al. Guidelines for reporting clinical features in cases with MECP2 mutations. Brain Dev 2001; 23: 208-211.

6. Amir RE, Van den Veyver IB, Schultz R, et al. Influence of mutation type and $\mathrm{X}$ chromosome inactivation on Rett syndrome phenotypes. Ann Neurol 2000; 47: 670-679.

7. Cuddapah VA, Pillai RB, Shekar KV, et al. MethylCpG-binding protein 2 (MECP2) mutation type is associated with disease severity in Rett syndrome. J Med Genet 2014; 51: 152-158.

8. Monros E, Armstrong J, Aibar E, Poo P, Canos I, Pineda M. Rett syndrome in Spain: mutation analysis and clinical correlations. Brain Dev 2001; 23: 251-253.

9. Amir RE, Van den Veyver IB, Wan M, Tran CQ, Francke $\mathrm{U}$, Zoghbi HY. Rett syndrome is caused by mutations in X-linked MECP2, encoding methyl-CpG-binding protein 2. Nat Genet 1999; 23: 185-188.

10. Adegbola AA, Gonzales ML, Chess A, LaSalle JM, Cox GF. A novel hypomorphic MECP2 point mutation is associated with a neuropsychiatric phenotype. Hum Genet 2009; 124: 615-623.

11. Neul JL, Fang P, Barrish J, et al. Specific mutations in methyl-CpG-binding protein 2 confer different severity in Rett syndrome. Neurology 2008; 70: 1313-1321.
12. Christodoulou J, Ho G. MECP2-Related Disorders. In: Pagon RA, Adam MP, Ardinger $\mathrm{HH}$, et al. (Eds). GeneReviews ${ }^{\circledR}$ (Updated 2012 Jun 28). Seattle: University of Washington, 2012: 1993-2017.

13. Christodoulou J, Grimm A, Maher T, Bennetts B RettBASE: The IRSA MECP2 variation database-a new mutation database in evolution. Hum Mutat 2003; 21: 466-472.

14. Gillberg C. Autism and Rett syndrome: some notes on differential diagnosis. Am J Med Genet 1986; 1: 127-131.

15. Marangi G, Zollino M. Pitt-Hopkins syndrome and differential diagnosis: a molecular and clinical challenge. J Pediatr Genet 2015; 4: 168-176.

16. Perry A, Sarlo-McGarvey N, Factor DC. Stress and family functioning in parents of girls with Rett syndrome. J Autism Dev Disord 1992; 22: 235-248.

17. Lambert L, Bienvenu $\mathrm{T}$, Allou L, et al. MEF2C mutations are a rare cause of Rett or severe Rett-like encephalopathies. Clin Genet 2012; 82: 499-501.

18. Allou L, Julia S, Amsallem D, et al. Rett-like phenotypes: expanding the genetic heterogeneity to the KCNA2 gene and first familial case of CDKL5-related disease. Clin Genet 2017; 91: 431-440.

19. Tarquinio DC, Hou W, Neul JL, et al. Age of diagnosis in Rett syndrome: patterns of recognition among diagnosticians and risk factors for late diagnosis. Pediatr Neurol 2015; 52: 585-591.

20. Bisgaard AM, Schönewolf-Greulich B, Ravn K, Ronde G. Is it possible to diagnose Rett syndrome before classical symptoms become obvious? Review of 24 Danish cases born between 2003 and 2012. Eur J Paediatr Neurol 2015; 19: 679-687.

21. Fehr S, Bebbington A, Ellaway C, Rowe P, Leonard $\mathrm{H}$, Downs J. Altered attainment of developmental milestones influences the age of diagnosis of rett syndrome. J Child Neurol 2011; 26: 980-987.

22. Einspieler C, Kerr AM, Prechtl HF. Is the early development of girls with Rett disorder really normal? Pediatr Res 2005; 57: 696-700.

23. Moeschler JB, Shevell M. Comprehensive evaluation of the child with intellectual disability or global developmental delays. Pediatrics 2014; 134: 903-918.

24. Kerr AM. Early clinical signs in the Rett disorder. Neuropediatrics 1995; 26: 67-71.

25. Lee JY, Leonard H, Piek JP, Downs J. Early development and regression in Rett syndrome. Clin Genet 2013; 84: $572-576$.

26. Ager S, Fyfe S, Christodoulou J, Jacoby P, Schmitt L, Leonard H. Predictors of scoliosis in Rett syndrome. J Child Neurol 2006; 21: 809-813.

27. Glaze DG, Percy AK, Skinner S, et al. Epilepsy and the natural history of Rett syndrome. Neurology 2010; 74: 909-912.

28. Weaving LS, Williamson SL, Bennetts B, et al. Effects of MECP2 mutation type, location and X-inactivation in modulating Rett syndrome phenotype. Am J Med Genet A 2003; 118: 103-114. 
29. Schanen C, Houwink EJ, Dorrani N, et al. Phenotypic manifestations of MECP2 mutations in classical and atypical Rett syndrome. Am J Med Genet A 2004; 126: 129-140.

30. Cheadle JP, Gill H, Fleming N, et al. Long-read sequence analysis of the MECP2 gene in Rett syndrome patients: correlation of disease severity with mutation type and location. Hum Mol Genet 2000; 9: 1119-1129.
31. Zoghbi HY, Percy AK, Schultz RJ, Fill C. Patterns of X chromosome inactivation in the Rett syndrome. Brain Dev 1990; 12: 131-135.

32.Archer H, Evans J, Leonard H, et al. Correlation between clinical severity in patients with Rett syndrome with a p.R168X or p.T158M MECP2 mutation, and the direction and degree of skewing of X-chromosome inactivation. J Med Genet 2007; 44: 148-152. 